\title{
INDEXIKALISCHE LEISTUNGEN DER MODALPARTIKELN UND IHRE NATÜRLICHKEITSTHEORETISCHE BEWERTUNG
}

\section{MODALPARTIKELN ALS PROTOTYPISCHE ERSCHEINUNG BESTIMMTER TEXTSORTEN}

Statistische Auszählungen zeigen, daß Modalpartikeln (Abtönungspartikeln) als eine prototypische Erscheinung bestimmter Textsorten angesehen werden können. Insgesamt läßt sich sagen, daß die Partikeln und selbst die zahlenmäßig kleine Funktionsklasse der Modalpartikeln in Texten erstaunlich oft vorkommen. $\mathrm{Zu}$ den 5000 in Kaedings Korpus häufigsten Wörtern gehören die Partikeln eben, denn, doch, jă, nur, schon, vielleicht (Meier 1978).

Der Hauptverwendungsbereich von Modalpartikeln (MPn) ist die umgangssprachliche natürliche Konversation (vgl. Franck 1980:24). Dies zeigen bereits Weydts Befunde (1969:99f.):

- MPn kommen häufiger in gesprochener Sprache vor und seltener in geschriebener Sprache,

- MPn treten häufiger in umgangssprachlicher-alltäglicher Sprache auf und seltener in gehobener Sprache,

- MPn erscheinen häufiger in spontaner Sprache und seltener in geplanter, vorformulierter oder ritueller Sprache,

- MPn sind häufiger in dialogischer Sprache und seltener in monologischer Sprache.

Aber auch innerhalb der mündlichen Kommunikation müssen Unterschiede in der Verwendung von MPn angenommen werden. Hentschel (1986:238ff) vermutet, daß zwischen dem Vorkommen von MPn und dem Privatheitsgrad eines Textes Zusammenhänge bestehen. Der Privatheitsgrad eines Textes kann mit Faktoren wie den folgenden bestimmt werden:

- dem Grad der Vertrautheit der Kommunikationsteilnehmer untereinander,

- dem Grad der Situationsvertrautheit,

- der persönlichen Betroffenheit durch das Gesprächsthema und Interesse am Gesprächsthema, 
- Differenzen im sozialen Rang der Teilnehmer,

- Vertrautheit des Kommunikationsortes und -mediums,

- Häufigkeit der Interaktion ('Lebhaftigkeit') und

- Arten des Gesprächsverlaufs (z.B. deskriptiv, argumentativ, assoziativ).

Die Häufigkeit, mit der MPn verwendet werden, steigt in dem Maße, wie die Kommunikation einen informellen, persönlichen, vertrauten und argumentativ-assoziativen Charakter annimmt, d.h. mit steigendem Privatheitsgrad (Hentschel 1986:243). Für Texte mit hohem Privatheitsgrad ist der Gebrauch von MPn also prototypisch, d.h. auch natürlich. Im Dialog Unterhaltung beim Morgenkaffee, einem Text mit hohem Privatheitsgrad, waren 5,13\% aller Textwörter MPn, in der Diskussion Schulklasse mit Präses Scharf, einem Text mit niedrigem Privatheitsgrad, hingegen nur 0,91\% aller Textwörter (beide Texte aus: Heutiges Deutsch 1975).

\section{MODALPARTIKELHALTIGE ÄUSSERUNGEN IM NATÜRLICHKEITSKONZEPT}

\subsection{GRUNDANNAHME}

In der Arbeitshypothese, derer ich mich bediene, wird die Existenz von einerseits verstärkten und entsprechenden nichtverstärkten syntaktischen Konstruktionen und andererseits die Existenz von geschwächten und entsprechenden nichtgeschwächten syntaktischen Konstruktionen postuliert.

Die Grundannahme besagt folgendes: Am Anfang (d.h. im Stadium, in welchem eine Konstruktion nur als eine syntaktische Variante eines anderen Ausdrucks zu werten und noch nicht grammatikalisiert ist) behaupten sich verstärkte Konstruktionen vorzugsweise unter relativ komplizierten (markierten) grammatischen Verhältnissen und verbreiten sich in ihrer späteren Entwicklung unter Umständen auch unter weniger komplizierten grammatischen Verhältnissen. Geschwächte Konstruktionen dagegen behaupten sich am Anfang vorzugsweise unter relativ einfachen (weniger markierten) grammatischen Verhältnissen und verbreiten sich später möglicherweise auch unter weniger einfachen grammatischen Verhältnissen (Näheres zum theoretischen Hintergrund ist in Orešnik/Snedec/Teržan/Trobevšek-Drobnak 1990 und in Orešnik $1990 \mathrm{zu}$ finden).

\subsection{SEMIOTISCHE GRUNDLAGEN}

Peirce (vgl. Dressler 1989:23, Nöth 1975:16ff) unterscheidet drei Stufen in der Objektrelation von Zeichen: das Ikon, den Index und das Symbol. In der Hierarchie der drei Zeichentypen steht das Symbol (als konventionelles Zeichen) am höchsten, denn die Herstellung der Objektrelation ist allein vom Interpreten des Zeichens abhängig. Diese Stufung der Zeichentypen entspricht also ihrem Arbitraritätsgrad. 
MPn können als indexikalische Ausdrücke angesehen werden. Wie alle sprachlichen Zeichen sind sie zugleich auch Symbole. Für MPn ist eine reduzierte Semantik charakteristisch, d.h. sie sind aus Lexemen mit voller referentieller (adjektivischer, adverbialer) oder expliziter synkategorematischer Bedeutung (Konjunktionen, Gradpartikeln, Steigerungspartikeln, Gliederungspartikeln) entstanden. Trotz ihrer reduzierten Semantik verfügen sie noch über einige gemeinsame Merkmale mit ihrem Ausgangswort. War das Ausgangswort schon deiktisch verwendbar, behielten die MPn die endophorische Verweisrichtung in der Regel bei (meist die anaphorische). Als neue Verweisrichtung kommt bei den MPn die exophorische hinzu, d.h. der Verweis auf die interaktionellen Beziehungen, auf die kommunikative Situation. Die exophorische Verweisrichtung wird bei den MPn zur dominierenden (und nach meiner Meinung markierten) Verweisrichtung. MPn sind sprachliche Symbole, die gleichzeitig Zeichenfunktionen von niedrigerer Semiotizität erfüllen. Solche Zeichen nennt Peirce degenerierte Zeichen (Nöth 1975:17).

Betrachten wir die drei Zeichentypen hinsichtlich ihrer Generalisierungskraft, dann ist es das Symobl, das auf der höchsten Stufe steht, während der Index auf der niedrigsten Stufe in der Zeichenhierarchie steht, denn nach Peirce ist der Index direkt von seinem Objekt abhängig, das deshalb auch in der Kommunikationssituation vorhanden sein muß. Das Ikon steht hinsichtlich seiner Generalisierungskraft zwischen Index und Symbol (Nöth 1975:19). Im Gegensatz zu Nöth 1975 sieht Peirce das Merkmal der Kontiguität als wesentliches Merkmal an, um Indices auf eine höhere Stufe in der Zeichenhierarchie zu stellen als das Ikon. Letzteres zeigt nämlich größere oder kleinere Übereinstimmungen mit seinem Objekt, während Indices keine Ähnlichkeit mit ihrem Objekt zu haben brauchen (vgl. auch Dressler 1989:13ff). Allein das Merkmal der Kontiguität sichert, daß ein Index mit seinem Objekt verbunden wird. Nöth 1975:19 betont, daß die Objektrelation beim Index wegen dessen perzeptueller Nähe zu seinem Objekt einfacher herzustellen ist. Dies läßt sich anhand der Genese des Zeichengebrauchs bei Kindern verdeutlichen, die nach Piaget 1962:163, 278 (Nöth 1975:19) durch zunehmende Differenzierung von Zeichen und Objekt gekennzeichnet ist. Am Anfang der Entwicklung steht eine pars-pro-toto-Relation, d.h. Zeichen (Indices) bestehen zunächst nur aus Teilen oder Teilaspekten des Objektes. Zeichen und Objekt sind in dieser Entwicklungsphase noch undifferenziert. Ein weiteres Argument dafür, daß Indices als einfachste Zeichen angesehen werden können, ist die Tatsache, daß indexikalischer Zeichengebrauch (mit wenigen Ausnahmen) der einzige Zeichengebrauch ist, der in verschiedenen Formen der Tierkommunikation vorkommt. Das Ikon impliziert hingegen eine stärkere Differenzierung von Zeichen und Objekt, denn es setzt eine geistige Umstrukturierung des Objektes voraus. Wenn man mit Nöth 1975:19 situationsbezogene Bedingungen der Zeichenhaftigkeit ebenfalls berücksichtigt, dann kann der Index nach dem Arbitraritätsgrad als Zeichen eingestuft werden, das in der Zeichenhierarchie am weitesten unten steht. 


\subsection{ARBEITSHYPOTHESE}

Zunächst habe ich angenommen, daß die folgenden Eigenschaften für die Hypothese sprechen könnten, eine MP-haltige Äußerung sei im Vergleich zu einer MPlosen propositionsgleichen Äußerung eine verstärkte Konstruktion:

1. Eine MP-haltige Äußerung ist nicht so zeit- oder platzsparend wie eine MPlose Äußerung, denn sie enthält eine zusätzliche syntaktische Stelle.

2. Eine MP-haltige Äußerung hat eine engere Bedeutung, denn durch das Hinzufügen der MP wird die Bedeutung des Äußerung genauer bestimmt bzw. modifiziert.

3. Dem Sprecher bereitet die Auswahl einer dem kommunikativen Sinn entsprechenden MP Schwierigkeiten.

4. Dem Hörer hingegen erleichtert die MP das Verständnis des kommunikativen Sinnes der Äußerung.

Historisch gesehen sind MPn (aufgrund ihrer reduzierten Semantik) in einfacheren grammatischen Umgebungen entstanden, wirken nun aber in den Äußerungen, in denen sie auftreten, als verstärkende Elemente auf der Beziehungsebene.

Anhand der Arbeitshypothese über die Komplexität von MP-Äußerungen habe ich Vorhersagen abgeleitet und diese mit Hilfe eines sprachlichen Korpus statistisch uiberprüft. Das sprachliche Korpus bestand aus den Telefongesprächen von Brons-Albert (1984) und den gesprochenen Texten des IdS (Freiburger Korpus in: Heutiges Deutsch 1975). Die 14 überpruiften Vorhersagen beschränken sich vor allem auf morphologische und syntaktische Eigenschaften MP-haltiger und MP-loser Äußerungen, und zwar auf die Satzlänge, die Äußerungslänge, auf die Struktur der Äußerung (d.h. Anzahl der Sätze einer Äußerung, Subordination von Sätzen), auf das Verhältnis zwischen Hauptund Nebensatz, auf das Verhältnis zwischen den Satzmodi, auf das Verhältnis zwischen affirmativen und negierten Sätzen, auf das Verhältnis der Sätze mit und ohne Satzklammer, auf das Verhältnis zwischen den Werten der einzelnen morphosyntaktischen Kategorien des Verbs (Person, Numerus, Tempus, Modus und Genus verbi). Die statistischen Ergebnisse sind in Petrič (1993) und in Petrič (1995) veröffentlicht und sollen hier nicht (detailliert) vorgeführt werden. Die Unterschiede zwischen Grundsample (mit MP-haltigen Äußerungen) und Kontrollsample sind in den folgenden Fällen signifikant (in Klammern stehen die Ausgangshypothesen):

1. Äußerungen, in denen ein MP-haltiger Satz auftritt, sind kürzer (Längere Äußerungen sind markierter).

2. Äußerungen, in denen ein MP-haltiger Satz auftritt, enthalten seltener einen Nebensatz (Äußerungen mit Hypotaxe sind markierter als Äußerungen mit Parataxe).

3. MPn treten häufiger in selbständigen oder abhängigen Hauptsätzen auf und seltener in Nebensätzen (Nebensätze sind markierter als Hauptsätze).

4. Der Anteil von Nicht-Aussagesätzen ist im Grundsample größer (Fragesätze, Imperativsätze, Wunschsätze und Exklamativsätze sind markierter als Aussagesätze). 
5. Der Anteil negierter Sätze ist im Grundsample größer (Negierte Sätze sind markierter als affirmative Sätze).

6. MP-haltige Sätze kommen häufiger ohne Satzklammer vor (Der Satzrahmen ist eine zweiteilige diskontinuierliche Konstruktion und ist im Vergleich zu einer einteiligen Konstruktion oder einer zweiteiligen kontinuierlichen Konstruktion markierter).

7. Der Anteil der dritten Person ist im Grundsample größer (Die erste und die zweite Person sind markierter als die dritte).

8. Der Anteil des Präsens ist im Grundsample größer (Die Vergangenheits- und Zukunftstempora sind markierter als das Präsens).

9. Der Anteil des Indikativ ist im Grundsample kleiner (Der Imperativ und der Konjunktiv sind markierter als der Indikativ).

Nur die Ergebnisse unter Punkt 4,5 und 9 entsprechen den aus den oben genannten Eigenschaften abgeleiteten Vorhersagen. Der Umstand, daß die meisten Vorhersagen nicht bestätigt wurden, soll in den folgenden Abschnitten kommentiert werden.

2.3.1 Nach meinen statistischen Ergebnissen ist es zu bezweifeln, ob der Platzoder Zeitverlust bei so kurzen Funktionswörtern wie den MPn eine wesentliche Rolle spielt. Relativiert werden muß auch die Behauptung, daß eine zusätzliche syntaktische Stelle für eine Zunahme der semantischen Komplexität einer Äußerung ausschlaggebend ist. MP-haltige Äußerungen enthalten zwar eine zusätzliche syntaktische Stelle (nämlich die MP, also eine Adverbposition), aber eine MP ist in ihrer semantischen Bedeutung so weit reduziert, daß die syntaktische Bereicherung der Äußerung keine semantische Bereicherung mit sich bringt.

2.3.2 Grundlegend ist die angemessene Bewertung der Bedeutung von MPn. Ich nehme an, daß eine MP-haltige Äußerung im Vergleich zu einer propositionsgleichen MP-losen Äußerung eine engere Bedeutung hat, denn durch das Hinzufügen der MP wird die Bedeutung der Äußerung genauer bestimmt bzw. modifiziert. Diese Behauptung stützt sich auf folgende Überlegung: MPn sind zwar semantisch (d.h. denotativ, referentiell, propositional) ausgebleicht, aber dennoch nicht bedeutungsleer. Die MP doch zum Beispiel verfügt über zumindest zwei Bedeutungskomponenten: über die der Anaphorizität und die des Gegensatzes. Bei MP-Kombinationen in einer Äußerung addieren sich die Bedeutungen der einzelnen MPn (Freges Kompositionsprinzip).

(1) Ich bin ja doch deine Mami.

(2) Komm doch mal!

Die Anzahl der Bedeutungskomponenten, die zum Satz durch MP-Kombinationen hinzugefügt werden, ist also noch größer, als dies beim Einsatz einer einzelnen MP der Fall war. Zu entsprechenden Ergebnissen kommen wir auch bei anderen MPn.

Einige unserer statistischen Ergebnisse zeigen eher Anhaltspunkte dafür, daß MPhaltige Äußerungen syntaktisch und semantisch einfacher sind als MP-lose Äußerungen (vgl. etwa die Ergebnisse unter den Punkten 1-3 und 6-8). Die semantischen Merkmale 
der MPn sind von so allgemeiner Natur, daß sich das Hinzufügen von MPn im Komplexitätsgrad der Äußerungsstruktur und Äußerungssemantik kaum niederschlägt. Ich habe den Eindruck, daß es eher umgekehrt ist, d.h. daß MP-haltige Äußerungen einfacher sind als MP-lose Äußerungen.

Es erhebt sich die Frage, ob die Hinzufügung von semantischen Merkmalen durch die MP dennoch eine Beschränkung für die MP-Äußerung zur Folge hat und ob man dennoch behaupten kann, daß MP-haltige Äußerungen (zumindest einige) Eigenschaften verstärkter Konstruktionen aufweisen. Eine Beschränkung für die MP-Äußerung ist vor allem in Bezug auf die Fortsetzungsmöglichkeiten im Text zu sehen, d.h. auf textlinguistischer Ebene. Da eine MP semantische Merkmale, wenn auch sehr unspezifische Merkmale, zur Äußerungsbedeutung beisteuert, sollte eine MP-Äußerung kontextuell eingeschränkter verwendbar sein als eine MP-lose Äußerung. Die Verwendung der MP-Äußerung auf bestimmte Umgebungen wird durch die indexikalische Kraft der MP eingeschränkt. Die MP ist dabei auf einen Vortext und einen Situationszusammenhang angewiesen, der seinen Gebrauch auslösen kann, und eröffnet ein bestimmtes Fortsetzungsraster bzw. bestimmte Fortsetzungsraster. Nach dem Kohärenzprinzip werden die Ausgangsbedingungen des ersten Sprechakts zu den Eingangsbedingungen des nächsten Sprechakts (Franck 1980:44). Der Sprecher hat immer eine beschränkte Anzahl von Fortsetzungsmöglichkeiten - vom Inhalt und vom Ausdruck her. Bei der Analyse von Gesprächssequenzen kann man sich etwa folgende Fragen stellen (Franck 1980:169d):

- Ist die MP-haltige Äußerung mit der Vorgängeräußerung verbunden oder ist sie kataphorisch zur Folgeäußerung ausgerichtet? (Die anaphorische Verweisrichtung kann man als unmarkiert ansehen, die kataphorische als markiert.)

- Wird durch die MP-haltige Äußerung eine bestimmte Präferenz bezüglich des Fortsetzungsrasters angezeigt? (z.B. rhetorische Fragen als Beispiel indirekter Sprechakte, die als markiert angesehen werden können; die MPn erleichtern die Dekodierung eines indirekten Sprechaktes)

- Welche Eigenschaften hat der vorhergehende Gesprächszug und welche hat der folgende Gesprächszug? Ist vor der MP-haltigen Äußerung Sprecherwechsel notwendig oder bestehen bestimmte syntaktische und/oder semantische Beschränkungen in der Vorgängeräußerung? Welche kommunikative Rolle hat der Vorgängerzug? (Macht ein Element Sprecherwechsel notwendig, dann kann man es als pragmatisch markiert ansehen)

Die grammatischen Parameter, die ich im statistischen Vergleich herangezogen habe, sind vor allem auf morphologische und syntaktische Eigenschaften von MP-haltigen Äußerungen beschränkt. Um die Analyse auf die Textebene auszuweiten, wäre es sinnvoll, statt einzelner Äußerungen ganze Gesprächssequenzen miteinander zu vergleichen. Analog könnten wir behaupten, daß eine MP-haltige Gesprächssequenz im Vergleich zu einer MP-losen Gesprächssequenz eine verstärkte Gesprächssequenz ist, denn der Zusammenhang zwischen den einzelnen Teilen einer Sequenz wird für den 
Adressaten hör- und/oder sichtbar markiert. Aufgrund dieser Annahme könnten bestimmte Vorhersagen für das Verhalten von MP-Äußerungen in Sequenzen abgeleitet werden: z.B.

- daß die meisten Gesprächssequenzen Äußerungen mit solchen MPn enthalten, die die MP-haltige Äußerung stärker mit der nächsten Äußerung verbinden (Die meisten MPn sind allerdings anaphorisch, daher wäre wohl eher eine stärkere Bindung an die vorangehenden Äußerungen zu erwarten);

- daß die meisten Gesprächssequenzen solche MP-Äußerungen enthalten, die eher initiativ und nicht reaktiv sind;

- daß die meisten Gesprächssequenzen solche MP-Äußerungen enthalten, die nur bestimmte Fortsetzungsmöglichkeiten vorschreiben oder sogar nur eine einzige;

- daß die meisten Gesprächssequenzen solche MP-Äußerungen enthalten, die ganz bestimmte Eingangsbedingungen verlangen oder sogar nur eine einzige;

- daß die meisten Gesprächssequenzen solche MP-Äußerungen enthalten, die Sprecherwechsel auslösen usw.

Diese Vorhersagen betreffen vor allem die endophorische Verweisrichtung von MPn.

2.3.3 Es ist zu bezweifeln, daß der Gebrauch von MPn eine wesentliche Kodierungsschwierigkeit für den Sprecher darstellen. Ich nehme an, daß sie zumindest für den erwachsenen (deutschen) Sprecher leicht zu produzieren sind, was mit ihrer reduzierten Semantik zusammenhängen könnte. Ein weiteres Indiz für die Einfachheit von MPn könnte die Tatsache sein, daß Sprecher MPn weniger bewußt verwenden (höherer Automatisierungsgrad) als sprachliche Mittel mit ausgeprägter referentieller semantischer Bedeutung, etwa die Autosemantika (Verben, Nomina). Eine andere Frage ist, ob der Erst- oder Zweitspracherwerb von MPn leicht ist. Man muß nämlich erst entsprechende pragmatische (kommunikative) Erfahrungen gemacht haben, um die MPn angemessen in verschiedenen Situationen und Textsorten einsetzen zu können. Es ist (zumindest mir) unklar, wie schwierig der MP-Erwerb ist. Lindner 1983 führt einige Belege an, in denen bereits 2-3-jährige Vorschulkinder zuerst in Dialogen MPn wie mal und denn verwenden, d.h. zwei der häufigsten deutschen MPn. Das wäre ein Anzeichen dafür, daß MPn ihrer Bedeutung nach keine komplizierten sprachlichen Zeichen sind; das würde auch mit der Vorstellung von MPn als indexikalischen Zeichen übereinstimmen. In der Natürlichkeitstheorie hält man ein Element, das sich auf den illokutiven Bereich bezieht, für markierter als ein Element, das sich auf den lokutiven Bereich bezieht (vgl. Dotter 1990). Das MP-Paradigma zeigt meiner Meinung nach, daß sich Markiertheitswerte im Leben eines Menschen, d.h. nicht nur im Kindesalter, sondern auch im Erwachsenenalter, verändern können und daß die MPn (als endo- und exophorische Indices) mit zunehmender kommunikativer Erfahrung des Individuums an Markiertheit verlieren. 
2.3.4 Dekodierung von MP-haltigen und MP-losen Äußerungen. Die oben angeführten Eigenschaften von Indices, zu denen auch die MPn gehören, sind ein Zeichen dafür, daß MPn recht einfache sprachliche Mittel sein müßten. Sie stehen auf der Stufe von Signalen, d.h. sie drücken nicht selbst bestimmte Beziehungen aus, sondern sie zeigen nur auf sie. Viele unserer statistischen Ergebnisse scheinen diese Vorstellung von der Einfachheit dieser sprachlichen Mittel zu stützen.

Die Verweisungsfunktion ist auch für Personalpronomina, Possessivpronomina oder Lokal- und Temporaladverbien charakteristisch. Modalpartikeln zeigen jedoch nicht auf ein Objekt in Raum und Zeit, sondern auf Beziehungen, die zwischen Objekten in Raum und Zeit bestehen, d.h genauer auf interaktionelle, interpersonale, illokutive und attitudinale Bestandteile der Kommunikationssituation. Hentschel 1986 hat diese Art des Verweises als metakommunikative Deixis bezeichnet.

Die Annahme, daß Modalpartikeln als indexikalische Ausdrücke einfache sprachliche Mittel sind, steht jedoch nicht in Einklang mit der weitverbreiteten Ansicht, daß Modalpartikeln eine beträchtliche Schwierigkeit darstellen, zumindest für DAF-Lerner und Kinder im Vorschulalter. Betrachtet man zunächst isolierte propositionsgleiche Äußerungen mit und ohne Modalpartikeln, kann man feststellen, daß man zur Interpretation einer MP-haltigen Äußerung eine bestimmte geistige Anstrengung machen muß, denn man muß sich eine Situation oder entsprechende Vorgängeräußerung vorstellen, in der die MP-haltige Äußerung verwendet werden kann. Die Tatsache, daß der Hörer zur Dekodierung der isolierten MP- ̈̈ußerung mehr Denkarbeit investieren muß, ist ein Merkmal geschwächter Konstruktionen. Eine isolierte MP-haltige Äußerung kann mit einer propositionsgleichen MP-losen Äußerung und ihrem Ko(n)text verglichen werden. In diesem Vergleich wäre die MP-haltige Äußerung als geschwächte Konstruktion aufzufassen, weil die MP auf abstrakte und generalisierende Weise all das zusammenfaßt, was im $\operatorname{Ko}(n)$ text ausgedrückt wird. Eine MP-haltige Äußerung müßte demnach eine Komplizierung für den Hörer darstellen. Bei einer MP-losen Äußerung entfällt das Problem, daß man sich eine geeignete Kommunikationssituation vorstellen muß, bis zu einem gewissen Grade, da diese in ihrem situationellen Gebrauch meist weniger spezifisch ist.

Da aber Äußerungen gewöhnlich nicht isoliert vorkommen, sondern eingebunden in einem Textzusammenhang, muß die Frage, ob Modalpartikeln einfache oder komplizierte sprachliche Mittel sind, auch noch von einer anderen Seite aus betrachtet werden. Vergleicht man nun einen Situationszusammenhang, in dem eine MP-haltige Äußerung auftritt, mit einem Situationszusammenhang, in dem eine MP-lose propositionsgleiche Äußerung in derselben Funktion auftritt, dann kann man meiner Meinung nach eher feststellen, daß die geistige Anstrengung bei der Dekodierung des kommunikativen Sinnes der Äußerung geringer wird, wenn die MP realisiert wird. Eine Situation braucht man sich gewöhnlich nicht vorzustellen, man befindet sich ja in ihr. Die Situation beseitigt bestimmte mögliche Lesarten der Äußerung. Auch die Interpretation der MP ist von dieser Monosemantisierung und Monopragmatisierung betroffen. MPn kön- 
nen von dieser Perspektive aus wohl eher als ein ökonomisches Mittel für die Indizierung und (Re)Aktualisierung von relevanten Kommunikationshintergründen angesehen werden. Mit einer MP signalisiert der Sprecher dem Hörer, daß dieser lokal relevante Kommunikationszusammenhänge erschließen soll, wobei in Texten meist nicht ausgedrückt wird, welche Zusammenhänge das sind, wohl aber werden diese durch den Ko(n)text nahegelegt.

(3) B: Lieb von dir, daß du an uns denkst! - Aber was anderes: Was macht ihr heute abend?
A: Em, wir haben noch nichts Festes vor!
B: Habt noch nichts Festes vor. Ich weiß o noch nich, was los is, nur, eh, um neun- zehn Uhr dreißig is $<$ doch $>$ so ein interessanter LeichtathletikWettkampf.
A: Ah so, ja dann kommt $<$ mal $>$ rüber! (BA 2)

(4) Odnosi med državama so $v$ tem trenutku res precej zapleteni. Na neki način gre za dialog gluhih, saj je pri težkih zunanjepolitičnih vprašanjih vedno vmešana tudi notranja politika in v tem hipu je to na italijanski strani močno izraženo. Prišlo je $<$ pač > do sprememb v italijanski politiki, do večjega vpliva skrajnih sil; ... (Tageszeitung DELO, 17.9.1994: 23)

Die Beziehungen zwischen den beiden Staaten sind im Augenblick wirklich sehr kompliziert. Irgendwie handelt es sich um einen von Taubheit geprägten Dialog, denn mit schwierigen außenpolitischen Fragen ist immer auch die Innenpolitik verbunden, und in diesem Augenblick ist dies auf italienischer Seite stark ausgeprägt. Es ist $<$ halt $>$ zu Veränderungen in der italienischen Politik gekommen, zu einem größeren Einfluß radikaler Kräfte; ...

(5) To brigado potrebujemo, imeli jo bomo tudi v prihodnje, čeprav si nekdo zelo prizadeva, da bi jo oblatil. Nekdo si <pač> želi, da pričnemo sankcije proti tej brigadi, da bi to uporabil v svoje politične namene.

(Tageszeitung DELO, 24.9.1994:2)

Diese Brigade benötigen wir und werden sie auch in Zukunft erhalten, obwohl jemand danach trachtet, sie in den Schmutz zu ziehen. Jemand wünscht sich <nun

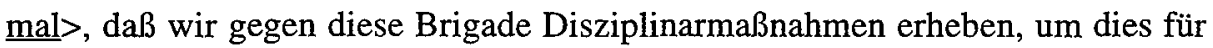
seine politischen Ziele auszunutzen.

MPn sind als sprachliche Indices zu bezeichnen, die dem Hörer auf ökonomische und unauffällige Weise argumentative Sprünge signalisieren (Abraham 1991:375; Krivonosov 1977, 1983). Nach dieser Annahme sind Modalpartikeln Signale (Signantia) für unvollständige Inferenzschritte in Texten. Allgemeinwissen wird häufig ausgespart, weil der Hörer es relativ leicht erschließen kann. Mit dem Gebrauch von MPn wird der Hörer in das pragmatische Wissen des Sprechers eingeweiht. Einige (Sprach)Kulturen machen von der Möglichkeit, unvollständige Textpassagen sichtbar zu machen, häufiger Gebrauch, andere weniger. 


\section{Komplexität und Markiertheit von Texten und MP-Gebrauch}

Die letzte These führt uns zurück zu einer bereits oben genannten, nämlich daß MPn häufiger in Dialogen auftreten, die einen höheren Privatheitsgrad aufweisen (Hentschel 1986). Die Tatsache, daß MPn häufiger in Texten mit Sprecherwechsel vorkommen, ist meiner Meinung nach ein weiteres Anzeichen dafür, daß MPn recht einfache sprachliche Zeichen sind, denn ich gehe von der Annahme aus, daß der Dialog die primäre Form der sprachlichen Kommunikation und der Monolog die sekundäre Form darstellt, d.h. daß der Dialog im Vergleich zum Monolog als unmarkierte sprachliche Form angesehen werden kann. Die Sprache dient vor allem der Verständigung zwischen Menschen, d.h. dem Informationstransport zwischen Kommunikationsteilnehmern, und im geringeren Maße der Verständigung eines Menschen mit sich selbst (Für letzteren Zweck wäre Sprache wohl überhaupt nicht notwendig). Der Transport von Informationen mit Hilfe eines semiotisch reichen Zeichensystems ermöglicht erst die Organisation und Koordinierung verschiedener menschlicher Tätigkeiten in einer Gemeinschaft. Der primäre Charakter des Dialogs wird daran sichtbar, daß auch der Monolog über dialogische Elemente verfügt: z.B. der Textproduzent versucht sich (bewußt oder unbewußt) in die Lage des Rezipienten zu versetzen (z.B. kann er Fragen stellen, die er dann selbst beantwortet). Darüber hinaus zeigt sich der primäre Charakter des Dialogs auch daran, daß selbst der Monolog immer einen Adressaten hat, der mit dem Textproduzenten nicht deckungsgleich ist. Sogar in sehr esotherischen Texten kann man davon ausgehen, daß der Textproduzent für einen Adressaten schreibt, nämlich für jemanden, der ähnlich denkt und fühlt wie der Textproduzent. Der primäre Charakter des Dialogs ist meiner Meinung nach auch daran erkennbar, daß die Anzahl von Sprechakten, die das verbale oder nonverbale Verhalten des Kommunikationsteilnehmers beeinflussen sollen (z.B. Warnung, Ratschlag, Vorschlag, Dank) wesentlich größer ist als die Anzahl von Sprechakten, die sich lediglich auf den Textproduzenten beziehen (z.B. Schimpfen, Überraschung, Resignation). Kinder erlernen ihre Muttersprache durch den Dialog, Selbstgespräche (z.B. beim Spielen) entwickeln sich oft erst auf einer späteren Entwicklungsstufe. Auch historisch gesehen sind viele monologische Textsorten jünger als die meisten dialogischen.

Aus der Unmarkiertheit des Dialogs folgt noch nicht, daß der Dialog als weniger komplizierte Kommunikationsform anzusehen wäre (In gewisser Weise ist der Unterschied vergleichbar mit dem zwischen Hauptsatz und Nebensatz: der Hauptsatz ist zwar strukturell komplizierter, der Nebensatz ist jedoch im Hinblick auf seinen Aufgabenbereich spezialisierter und kein prototypischer Satz, sondern selbst Teil eines Satzes). Aber der Dialog ist eine Kommunikationsform, die der menschlichen Kognition näher steht als der Monolog. Dafür kann man mehrere (psychologische und soziologische) Gründe verschiedener Art anführen: z.B. das Individuum will Anerkennung und Achtung in der Gemeinschaft finden, das Individuum will einen Beitrag zur Gesellschaft leisten, das Individuum will sein Wissen und das Wissen seines Partners über- 
prüfen, die Aufmerksamkeitsdauer und damit auch die Zuhörbereitschaft ist teilweise vom Arbeitsspeicher des Individuums abhängig u.a. Auf der Darstellungsebene kann man wohl kaum behaupten, die eine oder andere Kommunikationsform sei komplizierter, denn diese Art von Komplexität ist von den äußeren Umständen und den Teilnehmern abhängig. Im Hinblick auf die Beziehungsebene könnte man jedoch behaupten, daß der Dialog im Vergleich zum Monolog eine unkompliziertere Form ist, denn der Hauptunterschied zwischen beiden Formen ist die stärkere Berücksichtigung (lokal) relevanter Ziele des Adressaten und der Beziehungen zwischen Sender und Adressat. In einem Dialog sind Wissenskorrekturen und Diskursrichtungsänderungen prinzipiell jederzeit möglich. Während ein authentischer Dialog mit all seinen Zielen und Ergebnissen ja nicht vorgefertigt ist, sondern während des Verständigungsablaufs entsteht, ist für den Monolog grundsätzlich eine zeitliche Diskrepanz hinsichtlich der Ziele und Inhalte, die von Produzent und Rezipient angestrebt werden, charakteristisch. Wenn der Textproduzent einen monologischen Text bildet, dann ist zunächst nur er selbst der Adressat ('innerer Adressat'). Die Bewertung der Ziele und Inhalte des Textes sind vor allem von ihm abhängig, d.h. von seinen Vorstellungen über die Welt. In einer heterogen zusammengesetzten Gemeinschaft kann der Textproduzent jedoch nicht gleichzeitig den prototypischen Rezipienten darstellen, denn die Interessen, Ziele und angestrebten Inhalte der übrigen Mitglieder der Gemeinschaft können sich wesentlich von den seinen unterscheiden. Damit der Text auch von der Gemeinschaft anerkannt wird (d.h. außerhalb des Bewußtseins des Textproduzenten), benötigt der Produzent eines monologischen Textes auch einen 'äußeren Adressaten'.

Die MPn gehören zu den indexikalischen Zeichen, die auf der Beziehungsebene operieren. Sie treten häufiger in dialogischen Texten auf als in monologischen. Der Gebrauch dieser indexikalischen Zeichen ist demnach natürlicher (weniger markiert) in Texten, in denen es mehr Möglichkeiten gibt, den Kommunikationspartner, sein Wissen und die jeweiligen Beziehungen zwischen den Teilnehmern zu berücksichtigen, d.h. in Dialogen. In dieser Hinsicht bestehen allerdings zwischen dialogischen Textsorten auch beträchtliche Unterschiede. In Dialogen mit höherem Privatheitsgrad sind situative Hinweise häufiger als in Dialogen mit niedrigerem Privatheitsgrad. Ein weiterer Unterschied zwischen Dialog und Monolog ergibt sich aus der Begrenztheit des Arbeitsspeichers eines Gesprächsteilnehmers. In spontan entstehenden Dialogen ist der Zeitdruck wesentlich größer. Dies führt dazu, daß die Sprecher viele Dinge unausgedrückt lassen und sich mit Andeutungen begnügen oder sich auf die Erschließbarkeit implizierter Inhalte verlassen. In Dialogen mit höheren Privatheitsgrad und betont assoziativem Gesprächsablauf ist das besonders typisch. Das führt zu einem vermehrten Auftreten von MPn.

Zum Schluß sollen noch einige Annahmen über die Komplexität von Texten und ein Vorschlag zu deren Markiertheitsbewertung vorgeführt werden. Dabei gehe ich von Kriterien aus, die in der Literatur zur Unterscheidung verschiedener Textsorten etabliert worden sind (Heutiges Deutsch 1975). Unsere Grundannahme am Anfang des zweiten 
Abschnitts möchte ich für diesen Zweck folgendermaßen umformulieren: Verstärkte Konstruktionen behaupten sich vorzugsweise unter relativ komplizierten (markierten) grammatischen und/oder relativ komplizierten pragmatischen Verhältnissen und verbreiten sich in ihrer späteren Entwicklung unter Umständen auch unter weniger komplizierten grammatischen und/oder pragmatischen Verhältnissen. Geschwächte Konstruktionen dagegen behaupten sich am Anfang vorzugsweise unter relativ einfachen (weniger markierten) grammatischen und/oder relativ einfachen pragmatischen Verhältnissen und verbreiten sich später möglicherweise auch unter weniger einfachen grammatischen und/oder pragmatischen Verhältnissen.

Für eine verstärkte Konstruktion beispielsweise können dann die folgenden Behauptungen gemacht werden: Die sprachliche Kodierung einer Proposition, eines Teils einer Proposition oder der Ausdruck von Beziehungen zwischen den Kommunikationsteilnehmern kann in einer bestimmten Kommunikationssituation nur mit Schwierigkeiten mit der angestrebten Genauigkeit realisiert werden. Die Beschaffenheit der Kommunikationssituation bzw. der Textsorte erschwert dem Sender die Textproduktion: z.B.

- das Globalziel oder die angestrebten Teilziele der Teilnehmer (bestimmte Globalziele, z.B. jemanden zu etwas veranlassen, sind schwieriger zu erreichen als andere Globalziele, z.B. jemanden über etwas informieren, insbesondere dann, wenn die Absichten der Teilnehmer einander ausschließen);

- die Teilnehmerzahl (eine größere Teilnehmerzahl erschwert den Kommunikationsablauf);

- das Verhältnis zwischen den Teilnehmern (große Unterschiede im Alter der Teilnehmer, in der Ausbildung, Autoritätsgefälle zwischen den Teilnehmern, hinsichtlich der Vertrautheit bzw. der Bekanntheit der Teilnehmer);

- die Situationsvertrautheit (die Nichtvertrautheit mit einer Kommunikationssituation erschwert die Textproduktion);

- die Situationsdistanz (Situationsdistanz erleichtert die Textproduktion, Betroffenheit erschwert sie);

- das Kommunikationsmedium (kein Sichtkontakt, akustische Störungen, Einwegkommunikation);

- der Kommunikationsort (ein nicht vertrauter Ort kann sich störend auf die Textproduktion auswirken);

- der Öffentlichkeitsgrad (Kommunikation in der Öffentlichkeit erschwert die Textproduktion);

- die Art der Themenbehandlung (die argumentative oder explikative Themenbehandlung ist für den Textproduzenten schwieriger als als die assoziative oder deskriptive).

Die Merkmale der Kommunikationssituation, die die Textproduktion erschweren, stufe ich als markiert ein. Die Textproduktion wird durch die Markiertheit sprachlicher 
(grammatischer) Mittel und/oder durch die Markiertheit der Kommunikationssituation erschwert. Während ein für den Textproduzenten kompliziertes sprachliches (grammatisches) Mittel für den Textrezipienten Dekodierungshilfe bieten kann, ist eine für den Textproduzenten schwierige (nichtsprachliche) Kommunikationssituation prinzipiell auch für den Textrezipienten schwierig. MPn scheinen häufiger in solchen Textsorten aufzutreten, die aufgrund der meisten oben angeführten Textkonstellationskriterien als unmarkierte Texte eingestuft werden könnten.

\section{ZUSAMMENFASSUNG}

Die Ergebnisse im zweiten Abschnitt zeigen, daß MP-haltige Äußerungen nur in ganz bestimmten Aspekten als verstärkte Konstruktionen angesehen werden können, und zwar in bezug auf ihre illokutiven und attitudinalen Funktionen, d.h. in bezug auf die Funktionen der MPn, als Indikatoren für bestimmte Sprechhandlungen und als Regulatoren für bestimmte Einstellungen dienen zu können.

Wenn sprachliche Ausdrücke semantische Merkmale verlieren, dann verhalten sie sich wie geschwächte Formen bzw. Konstruktionen. Sie geraten in einfache grammatische Umgebungen. Hierbei handelt es sich um Markiertheitsabbau auf morphologischsyntaktischer Ebene. Indexikalische Ausdrücke wie die deutschen MPn zeigen jedoch, $\mathrm{da} ß$ auf einer anderen Ebene als der Darstellungsebene, d.h. auf der Beziehungsebene, sprachliche Elemente auch an Markiertheit gewinnen können. Die statistischen Ergebnisse zeigen eine Affinität der MPn zu modalen Kategorien und lassen meiner Meinung nach die Interpretation $z u, d a ß ~ M P n$ in illokutiv markierten grammatischen Umgebungen auftreten. Die MPn wären demnach ein Fall von Markiertheitsabbau und Markiertheitsaufbau zugleich, obwohl auf verschiedenen sprachlichen Ebenen.

MPn sind als sprachliche Indices zu bezeichnen, die dem Hörer auf ökonomische und unauffällige Weise argumentative Sprünge signalisieren. Der Gebrauch dieser indexikalischen Zeichen ist demnach natürlicher (weniger markiert) in Texten, in denen es mehr Möglichkeiten gibt, den Kommunikationspartner, sein Wissen und die jeweiligen Beziehungen zwischen den Teilnehmern zu berücksichtigen, d.h. in Dialogen, insbesondere in solchen mit höherem Privatheitsgrad.

\section{LITERATUR}

Abraham, Werner: The Grammatization of the German Modal Particles. In: CLOSS TRAUGOTT, Elizabeth / HEINE, Bernd (eds.): Approaches to Grammaticalization. Volume I and II. Amsterdam, Philadelphia 1991. Volume II. S. 331-380.

Brons-Albert, Ruth: Gesprochenes Standarddeutsch. Telefondialoge. Studien zur deutschen Grammatik 18. Tübingen 1984. 
Dotter, Franz: Nichtarbitrarität und Ikonizität in der Syntax. Hamburg 1990.

Dressler, Wolfgang Ulrich: Semiotische Parameter einer textlinguistischen Natürlichkeitstheorie. Wien 1989.

Franck, Dorothea: Grammatik und Konversation. Königstein/Ts. 1980.

Hentschel, Elke: Funktion und Geschichte deutscher Partikeln. Ja, doch, halt und eben.

Tübingen 1986.

Heutiges Deutsch: Texte gesprochener deutscher Standardsprache II/3. IDS Mannheim

Forschungsstelle Freiburg i. Br. München, Düsseldorf 1975.

Krivonosov, Aleksej T.: Deutsche Modalpartikeln im System der unflektierbaren

Wortklassen. In: WEYDT, Harald (Hg.): Aspekte der Modalpartikeln - Studien zur deutschen Abtönung. Tübingen 1977. S. 176-216.

Krivonosov, Aleksej T.: Zur Rolle der Partikeln bei der Einsparung des Sprachmaterials.

In: WEYDT, Harald (Hg.) Partikeln und Interaktion. Tübingen 1983. S. 40-45.

Lindner, Katrin: Sprachliches Handeln bei Vorschulkindern. Tübingen 1983.

Meier, Helmut: Deutsche Sprachstatistik. Hildesheim, New York 1978.

Nöth, Winfried: Semiotik. Eine Einführung mit Beispielen für Reklameanalysen.

Tübingen 1975.

Orešnik, Janez: Periphrasen sind verstärkte Konstruktionen. In: Boretzky N./Enninger

W./ Stolz T. (Hgg.): Spielarten der Natürlichkeit - Spielarten der Ökonomie.

Beiträge zum 5. Essener Kolloquium über "Grammatikalisierung: Natürlichkeit und Systemökonomie" vom 6.10.-8.10.1988 an der Universität Essen. Bochum 1990. Zweiter Band, erster Halbband, S. 85-99.

Orešnik, Janez/Snedec, Andrej/Teržan, Karmen/Trobevšek-Drobnak, Frančiška: Introduction to the Subsequent Three Papers in the Present Volume. In: Linguistica XXX. Ljubljana 1990. S. 5-12.

Petrič, Teodor: Stavki z naklonskimi členki kot okrepljene skladenjske zgradbe $<$ Modalpartikelhaltige Sätze als verstärkte syntaktische Konstruktionen>. In: Jezik Tako in drugače. Zbornik prvega kongresa Društva za uporabno jezikoslovje Slovenije. Ljubljana 1993. S. 252-262.

Petrič, Teodor: Modalpartikeln und Natürlichkeitstheorie. Erscheint voraussichtlich 1995 im Sammelband mit den Referaten zum Symposion 'Natürlichkeitstheorie und Sprachwandel', abgehalten in Maribor, vom 13.-15. Mai 1993. WEYDT, Harald (1969): Abtönungspartikel. Homburg, Berlin, Zürich.

\section{Zusammenfassung}

Modalpartikeln (MPn) können als prototypische Erscheinung von Texten mit hohem Privatheitsgrad angesehen werden. Sie sind somit keine konstitutiven Elemente sondern regulative Elemente von Texten. Eine Differenzierung von Textsorten bezüglich ihrer Komplexität ist sowohl vom wissenschaftlichen als auch vom pädagogischen Standpunkt anzustreben. Ein möglicher Ansatzpunkt ist die Betrachtung von mikro- und mediostrukturellen Mitteln, welche die Textproduktion oder die Textrezeption erschweren bzw. erleichtern. Die zentrale Frage, die im Aufsatz erörtert wird, betrifft die Komplexität bzw. Einfachheit von MPn und MP-halltigen Äußerungen. Im Vergleich zu MP-losen Äußerungen können MP-haltige Äußerungen als verstärkte Konstruktionen ('markiert') oder geschwächte Konstruktionen ('unmarkiert') bewertet werden. Verstärkte 
Konstruktionen sind sie mit Bezug auf die (pragmatische) Funktion der MPn, denn als indexikalische sprachliche Zeichen helfen MPn dem Adressaten auf sehr ökonomische Weise beim Dekodieren von lokal relevanten Kommunikationshintergründen. Geschwächte Konstruktionen sind sie in bezug auf ihre semantische (denotative, referentielle) Einfachheit.

\section{Povzetek}

\section{KAZALNE LASTNOSTI NAKLONSKIH ČLENKOV IN NJIHOVO VREDNOTENJE V TEORIJI NARAVNOSTI}

Za naklonske členke je značilno, da pogosteje nastopajo $v$ besedilih $z$ visoko stopnjo zasebnosti. Potemtakem ne sodijo med konstitutivne besedilne prvine, temveč med regulativne, ki povečujejo variantnost besedilne vrste. Razlikovanje besedilnih zvrsti po njihovi zapletenosti je zaželeno iz znanstvenih in pedagoških razlogov. Možno izhodičče je opazovanje mikro- in mediostrukturnih sredstev, ki tvorbo ali razumevanje besedila otežujejo oz. olajšujejo. Osrednja tema prispevka je zapletenost oz. preprostost naklonskih členkov in povedi z naklonskimi členki. Domnevam, da imajo povedi z naklonskimi členki v primerjavi s povedmi, ki naklonskih členkov ne vsebujejo, nekatere lastnosti okrepljenih zgradb ('zaznamovanih') in nekatere lastnosti ošibljenih zgradb. Glede na (pragmatično) vlogo naklonskih členkov so povedi s temi členki okrepljene zgradbe, saj so naklonski členki indeksikalni znaki, ki naslovniku na zelo gospodaren način pomagajo razvozlati trenutno pomembno ozadje sporočila. Glede na pomensko (tj. denotativno, referencialno) preprostost naklonskih členkov so take povedi ošibljene zgradbe. 